\title{
Molecular Self-Assembly in a Family of Oxo-Bridged Dinuclear Ruthenium(IV) Systems
}

\author{
Marta Orts-Arroyo, Isabel Castro, ${ }^{*}$ Francesc Lloret, and José Martínez-Lillo* \\ Instituto de Ciencia Molecular (ICMol), Universitat de València, c/ Catedrático José Beltrán 2, \\ 4698o, Paterna, València, Spain
}

Table of contents

page

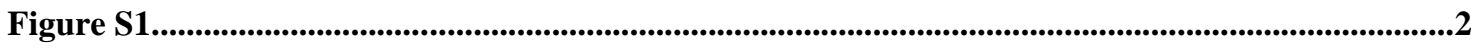

Figure S2

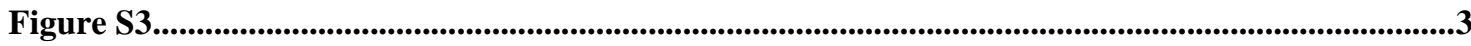

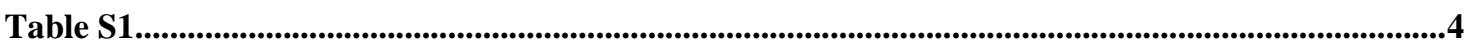

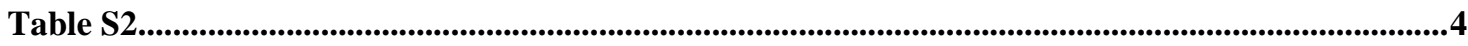

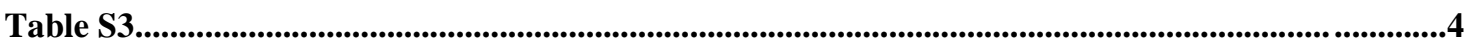

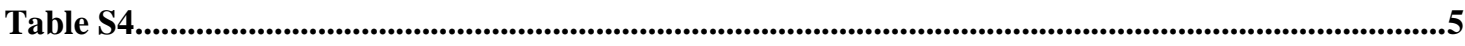

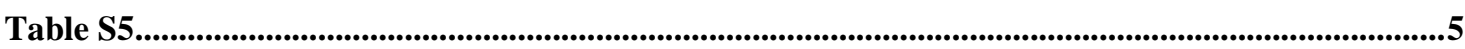

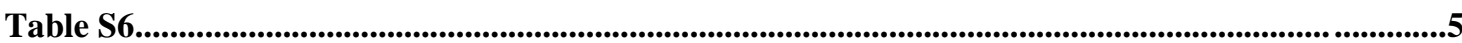

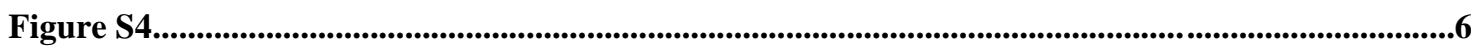

Figure S5.....................................................................................................................................................................6

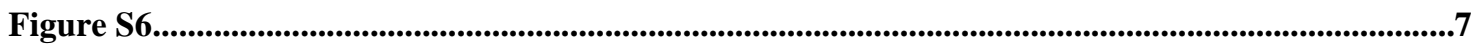

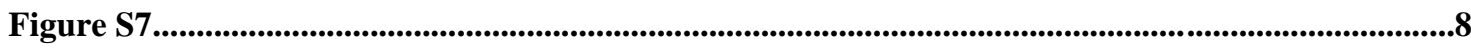

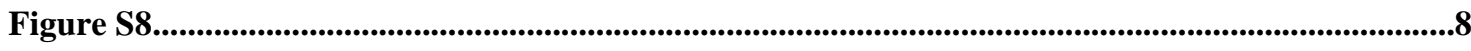

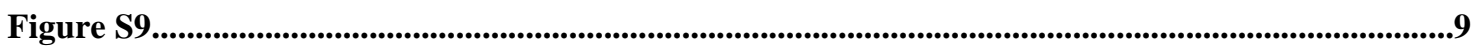

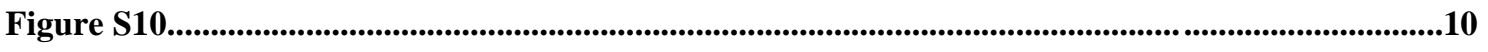

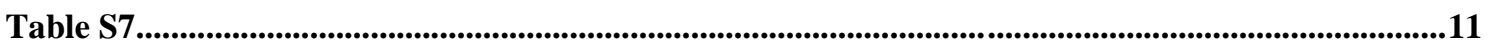

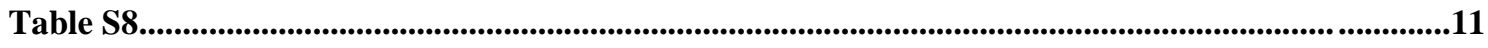

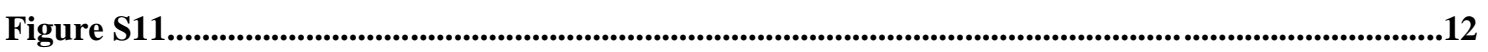

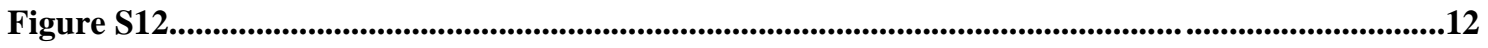




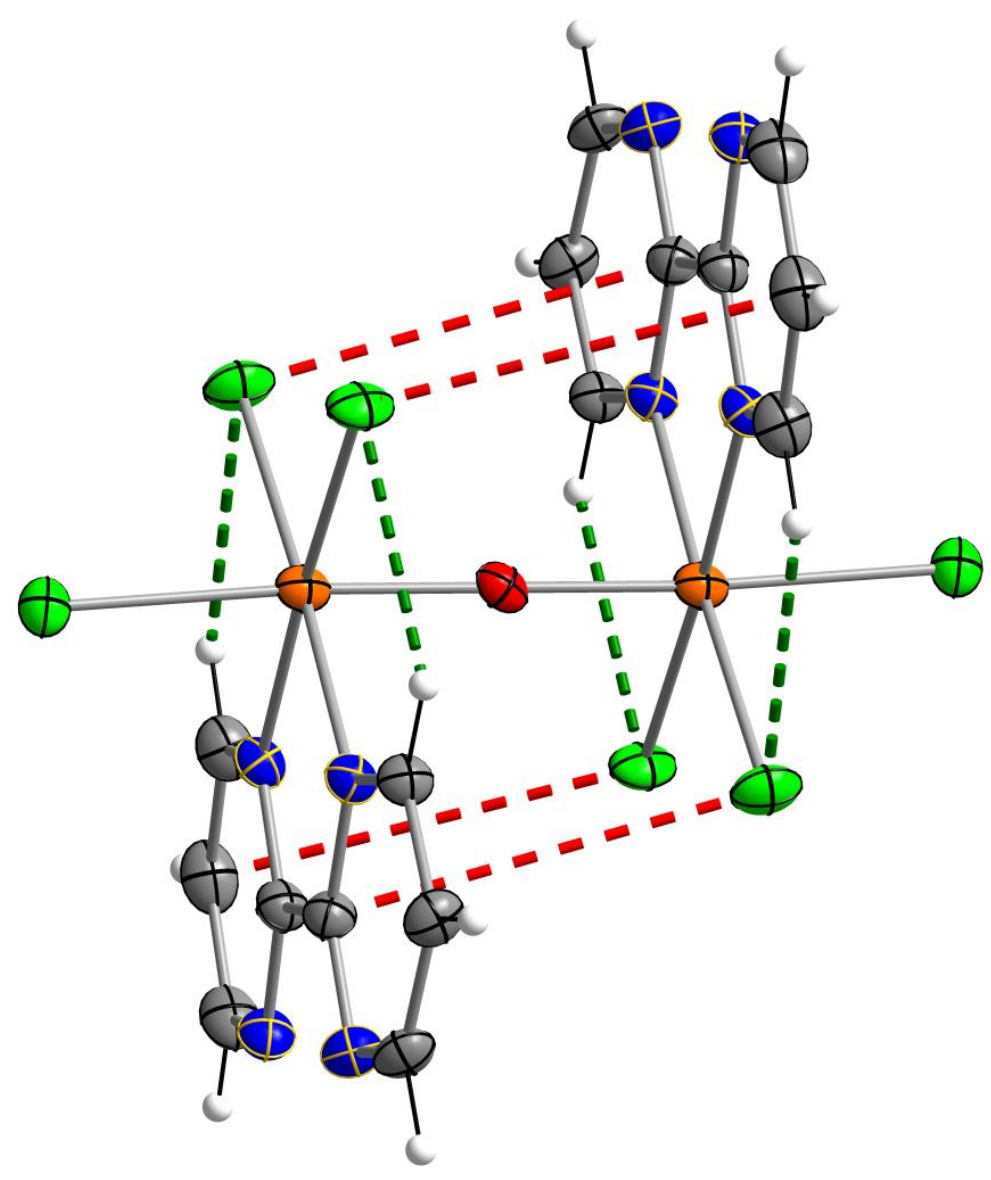

Figure S1. Detail of the intramolecular $\mathrm{Cl} \cdots \pi$ (dashed red lines) and $\mathrm{Cl} \cdots \mathrm{H}-\mathrm{C}$ (dashed green lines) interactions that occur in the dinuclear $\left[\left\{\mathrm{RuCl}_{3}(\mathrm{bpym})\right\}_{2}(\mu-\mathrm{O})\right]$ complex of 6. Thermal ellipsoids are depicted at the $50 \%$ probability level. Crystallization water molecules have been omitted for clarity. Color code: orange, $\mathrm{Ru}$; green, $\mathrm{Cl}$; red, $\mathrm{O}$; blue, $\mathrm{N}$; grey, C; white, $\mathrm{H}$. 


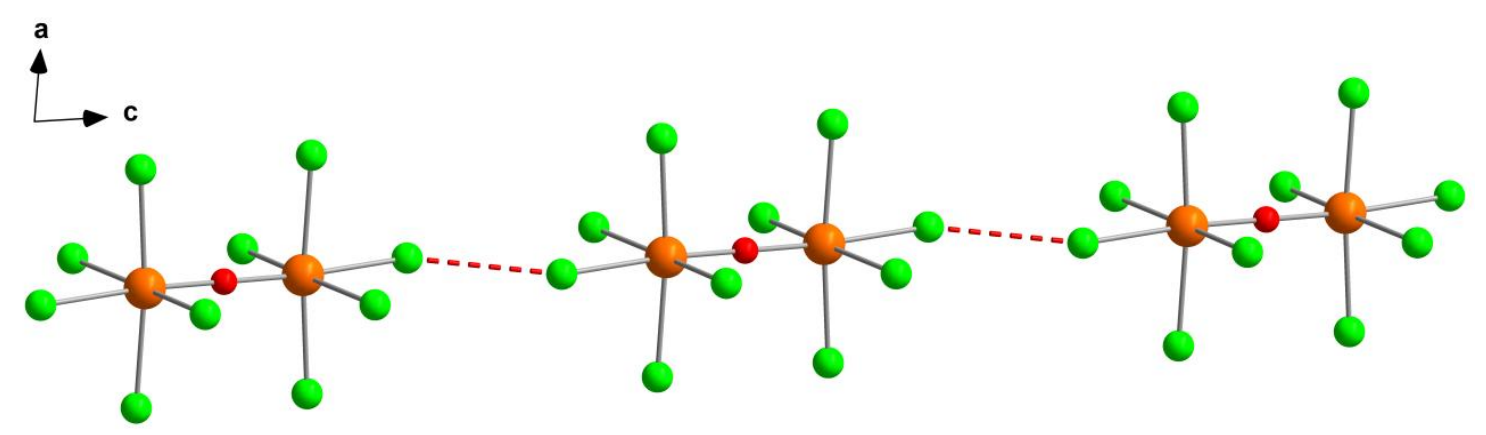

Figure S2. Detail of the one-dimensional motif generated by intermolecular $\mathrm{Cl} \cdots \mathrm{Cl}$ contacts (dashed red lines) between neighboring $\left[\left\{\mathrm{RuCl}_{5}\right\}_{2}(\mu-\mathrm{O})\right]^{4-}$ complexes in the crystal of 1. Color code: orange, $\mathrm{Ru}$; green, $\mathrm{Cl}$; red, $\mathrm{O}$.

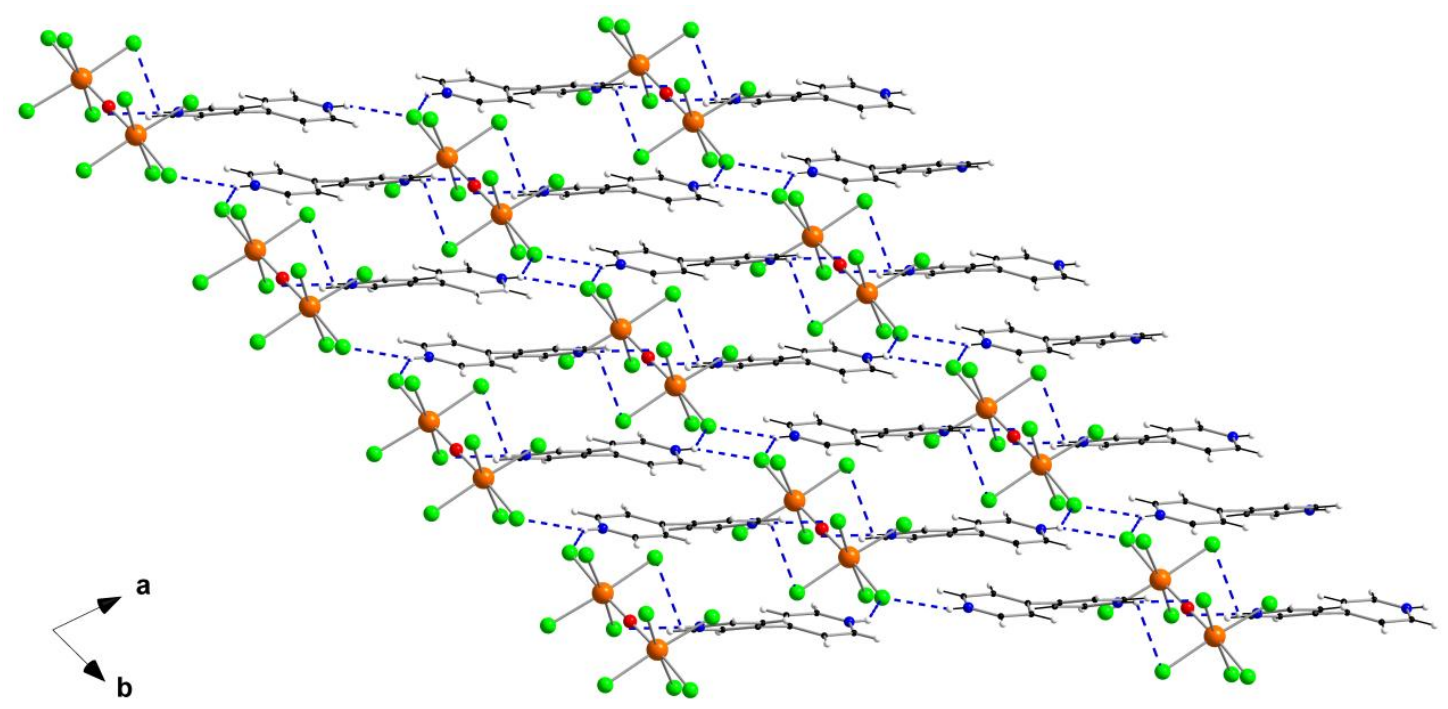

Figure S3. Perspective view along the $c$-axis direction of the two-dimensional motif formed by means of bifurcated three-centered hydrogen bonds (dashed blue lines) between $\left(\mathrm{H}_{2} \mathrm{bpy}\right)^{2+}$ cations and $\left[\left\{\mathrm{RuCl}_{5}\right\}_{2}(\mu-\mathrm{O})\right]^{4-}$ anions in the crystal of $\mathbf{1}$. Color code: orange, $\mathrm{Ru}$; green, $\mathrm{Cl}$; red, $\mathrm{O}$; blue, $\mathrm{N}$; black, $\mathrm{C}$; white, $\mathrm{H}$. 
Table S1. Values of the $\mathrm{C}-\mathrm{H} \cdots \mathrm{Cl}$ interactions in compound 1.

$\begin{array}{lrlll}\mathbf{C}-\mathbf{H} \cdots \mathbf{C l} & \mathbf{C}-\mathbf{H} / \mathbf{A} & \mathbf{H} \cdots \mathbf{C l} / \mathbf{A} & \mathbf{C} \cdots \mathbf{C l} / \mathbf{A}(\mathbf{C H C l}) /^{\circ} \\ & & & & \\ \mathrm{C}(1)-\mathrm{H}(1 \mathrm{C}) \cdots \mathrm{Cl}(3 \mathrm{~b}) & 0.930 & 2.95(1) & 3.579(1) & 125.9 \\ \mathrm{C}(1)-\mathrm{H}(1 \mathrm{C}) \cdots \mathrm{Cl}(3 \mathrm{~d}) & 0.930 & 2.90(1) & 3.393(1) & 114.7 \\ \mathrm{C}(4)-\mathrm{H}(4 \mathrm{~A}) \cdots \mathrm{Cl}(4 \mathrm{f}) & 0.930 & 2.91(1) & 3.477(1) & 121.0 \\ \mathrm{C}(5)-\mathrm{H}(5 \mathrm{~A}) \cdots \mathrm{Cl}(2 \mathrm{~g}) & 0.930 & 2.84(1) & 3.700(1) & 155.2 \\ \mathrm{C}(5)-\mathrm{H}(5 \mathrm{~A}) \cdots \mathrm{Cl}(4 \mathrm{f}) & 0.930 & 2.79(1) & 3.419(1) & 126.2 \\ \mathrm{C}(8)-\mathrm{H}(8 \mathrm{~A}) \cdots \mathrm{Cl}(4 \mathrm{e}) & 0.930 & 2.55(1) & 3.468(1) & 168.1 \\ \mathrm{C}(8)-\mathrm{H}(8 \mathrm{~A}) \cdots \mathrm{Cl}(5 \mathrm{e}) & 0.930 & 2.93(1) & 3.458(1) & 117.5 \\ \mathrm{C}(9)-\mathrm{H}(9 \mathrm{~A}) \cdots \mathrm{Cl}(1 \mathrm{c}) & 0.930 & 2.89(1) & 3.791(1) & 163.1 \\ \mathrm{C}(9)-\mathrm{H}(9 \mathrm{~A}) \cdots \mathrm{Cl}(2 \mathrm{c}) & 0.930 & 2.81(1) & 3.425(1) & 124.5 \\ \mathrm{C}(10)-\mathrm{H}(10 \mathrm{~A}) \cdots \mathrm{Cl}(1 \mathrm{f}) & 0.930 & 2.84(1) & 3.334(1) & 114.6\end{array}$

${ }^{a}$ Symmetry codes: $(\mathrm{b})=-\mathrm{x}+1,-\mathrm{y},-\mathrm{z}+1 ;(\mathrm{c})=\mathrm{x}+1, \mathrm{y}, \mathrm{z} ;(\mathrm{d})=\mathrm{x}, \mathrm{y}-1, \mathrm{z} ;(\mathrm{e})=-\mathrm{x}+2,-\mathrm{y},-\mathrm{z}+1$;

(f) $=-x+2,-y,-z$.

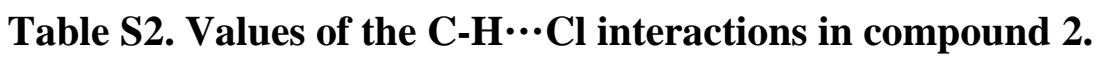

$\begin{array}{lllll}\mathbf{C}-\mathbf{H} \cdots \mathbf{C l} & \mathbf{C}-\mathbf{H} / \AA & \mathbf{H} \cdots \mathbf{C l} / \AA & \mathbf{C} \cdots \mathbf{C l} / \mathbf{A} & (\mathbf{C H C l}) /^{\circ} \\ & & & & \\ \mathrm{C}(18)-\mathrm{H}(18 \mathrm{~A}) \cdots \mathrm{Cl}(4) & 0.950 & 2.81(1) & 3.578(1) & 138.7 \\ \mathrm{C}(20)-\mathrm{H}(20 \mathrm{~A}) \cdots \mathrm{Cl}(1) & 0.950 & 2.81(1) & 3.727(1) & 163.3 \\ \mathrm{C}(30)-\mathrm{H}(30 \mathrm{~A}) \cdots \mathrm{Cl}(5 \mathrm{~d}) & 0.950 & 2.82(1) & 3.672(1) & 149.7 \\ \mathrm{C}(32)-\mathrm{H}(32 \mathrm{~A}) \cdots \mathrm{Cl}(8) & 0.950 & 2.95(1) & 3.610(1) & 127.8\end{array}$

${ }^{a}$ Symmetry codes: $(\mathrm{d})=-\mathrm{x}+1,-\mathrm{y},-\mathrm{z}+1$.

Table S3. Values of the $\mathrm{C}-\mathrm{H} \cdots \mathrm{Cl}$ interactions in compound 3.

$\begin{array}{lllll}\mathbf{C}-\mathbf{H} \cdots \mathbf{C l} & \mathbf{C - H} / \AA & \mathbf{H} \cdots \mathbf{C l} / \mathbf{A} & \mathbf{C} \cdots \mathbf{C l} / \AA & (\mathbf{C H C l}){ }^{\circ} \\ & & & & \\ \mathrm{C}(20)-\mathrm{H}(20) \cdots \mathrm{Cl}(2 \mathrm{a}) & 0.950 & 2.72(1) & 3.666(1) & 178.2 \\ \mathrm{C}(22)-\mathrm{H}(22) \cdots \mathrm{Cl}(3 \mathrm{a}) & 0.950 & 2.85(1) & 3.664(1) & 144.5 \\ \mathrm{C}(2)-\mathrm{H}(2 \mathrm{~A}) \cdots \mathrm{Cl}(2 \mathrm{~b}) & 0.980 & 2.95(1) & 3.848(1) & 152.6 \\ \mathrm{C}(4)-\mathrm{H}(4) \cdots \mathrm{Cl}(1 \mathrm{~b}) & 0.950 & 2.57(1) & 3.331(1) & 137.5 \\ \mathrm{C}(16)-\mathrm{H}(16) \cdots \mathrm{Cl}(4 \mathrm{~d}) & 0.950 & 2.96(1) & 3.514(1) & 118.7 \\ \mathrm{C}(26)-\mathrm{H}(26) \cdots \mathrm{Cl}(3 \mathrm{e}) & 0.950 & 2.78(1) & 3.631(1) & 150.0\end{array}$

${ }^{a}$ Symmetry codes: $(a)=-x+1,-y+2,-z+1 ;(b)=x,-y+3 / 2, z+1 / 2 ;(d)=x+1,-y+3 / 2, z+1 / 2 ;(e)=-x+1, y-$ $1 / 2,-\mathrm{z}+3 / 2$. 
Table S4. Values of the $\mathrm{C}-\mathrm{H} \cdots \mathrm{Cl}$ interactions in compound 4.

$\begin{array}{lllll}\mathbf{C}-\mathbf{H} \cdots \mathbf{C l} & \mathbf{C}-\mathbf{H} / \mathbf{A} & \mathbf{H} \cdots \mathbf{C l} / \mathbf{A} & \mathbf{C} \cdots \mathbf{C l} / \mathbf{A} & (\mathbf{C H C l}) /^{\circ} \\ & & & & \\ \mathrm{C}(1)-\mathrm{H}(1) \cdots \mathrm{Cl}(1 \mathrm{a}) & 0.950 & 2.75(1) & 3.228(1) & 112.0 \\ \mathrm{C}(3)-\mathrm{H}(3 \mathrm{~A}) \cdots \mathrm{Cl}(1 \mathrm{~b}) & 0.980 & 2.94(1) & 3.869(1) & 158.3 \\ \mathrm{C}(3)-\mathrm{H}(3 \mathrm{~A}) \cdots \mathrm{Cl}(3 \mathrm{~b}) & 0.980 & 2.80(1) & 3.411(1) & 121.1 \\ \mathrm{C}(5)-\mathrm{H}(5) \cdots \mathrm{Cl}(3 \mathrm{a}) & 0.950 & 2.69(1) & 3.393(1) & 131.5 \\ \mathrm{C}(21)-\mathrm{H}(21) \cdots \mathrm{Cl}(1 \mathrm{~b}) & 0.950 & 2.94(1) & 3.881(1) & 172.5 \\ \mathrm{C}(23)-\mathrm{H}(23) \cdots \mathrm{Cl}(2 \mathrm{~b}) & 0.950 & 2.84(1) & 3.602(1) & 137.6 \\ \mathrm{C}(27)-\mathrm{H}(27) \cdots \mathrm{Cl}(2) & 0.950 & 2.98(1) & 3.867(1) & 155.7\end{array}$

a Symmetry codes: (a) $=-x+1,-y,-z+1,(b)=x,-y-1 / 2, z+1 / 2$.

Table S5. Values of the $\mathrm{C}-\mathrm{H} \cdots \mathrm{Cl}$ interactions in compound 5.

$\begin{array}{lllll}\mathbf{C}-\mathbf{H} \cdots \mathbf{C l} & \mathbf{C - H} / \mathbf{A} & \mathbf{H} \cdots \mathbf{C l} / \mathbf{A} & \mathbf{C} \cdots \mathbf{C l} / \mathbf{A} & (\mathbf{C H C l}) /^{\circ} \\ & & & & \\ \mathrm{C}(7)-\mathrm{H}(7) \cdots \mathrm{Cl}(4 \mathrm{c}) & 0.950 & 2.99(1) & 3.815(1) & 146.5 \\ \mathrm{C}(17)-\mathrm{H}(17) \cdots \mathrm{Cl}(1 \mathrm{~b}) & 0.950 & 2.68(1) & 3.598(1) & 162.9\end{array}$

${ }^{\mathrm{a} S y m m e t r y ~ c o d e s: ~}(\mathrm{~b})=-\mathrm{x}+1,-\mathrm{y}+2,-\mathrm{z},(\mathrm{c})=\mathrm{x}, \mathrm{y}-1, \mathrm{z}$.

Table S6. Values of the C-H $\cdots \mathrm{Cl}$ interactions in compound 6.

$\begin{array}{lllll}\mathbf{C - H} \cdots \mathbf{C l} & \mathbf{C - H} / \mathbf{A} & \mathbf{H} \cdots \mathbf{C l} / \AA & \mathbf{C} \cdots \mathbf{C l} / \AA & (\mathbf{C H C l}) /^{\circ} \\ \mathrm{C}(1)-\mathrm{H}(1 \mathrm{~B}) \cdots \mathrm{Cl}(2) & 0.950 & 2.79(1) & 3.380(1) & 121.0 \\ \mathrm{C}(1)-\mathrm{H}(1 \mathrm{~B}) \cdots \mathrm{O}(2 \mathrm{wd}) & 0.950 & 2.56(1) & 3.363(1) & 143.1 \\ \mathrm{C}(2)-\mathrm{H}(2 \mathrm{~A}) \cdots \mathrm{Cl}(1 \mathrm{~g}) & 0.950 & 2.89(1) & 3.832(1) & 174.1 \\ \mathrm{C}(3)-\mathrm{H}(3 \mathrm{~B}) \cdots \mathrm{Cl}(1 \mathrm{~d}) & 0.950 & 2.89(1) & 3.717(1) & 146.8 \\ \mathrm{C}(6)-\mathrm{H}(6 \mathrm{~A}) \cdots \mathrm{Cl}(3) & 0.950 & 2.73(1) & 3.332(1) & 122.3 \\ \mathrm{C}(6)-\mathrm{H}(6 \mathrm{~A}) \cdots \mathrm{Cl}(3 \mathrm{~b}) & 0.950 & 2.77(1) & 3.584(1) & 144.7\end{array}$

${ }^{a}$ Symmetry codes: $(\mathrm{b})=-\mathrm{x},-\mathrm{y}+1,-\mathrm{z}+2 ;(\mathrm{d})=-\mathrm{x}+2,-\mathrm{y}+1,-\mathrm{z}+1 ;(\mathrm{g})=\mathrm{x}+1, \mathrm{y}, \mathrm{z}$. 


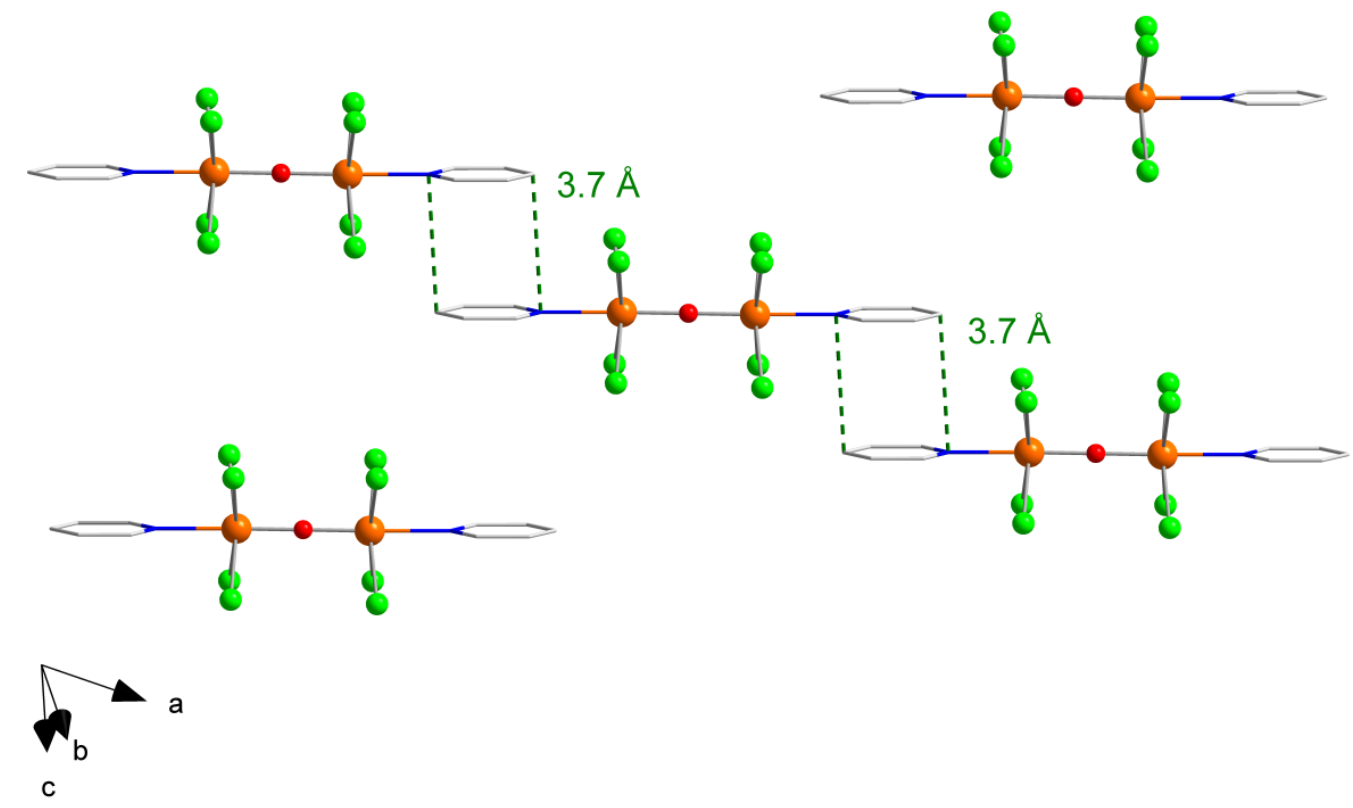

Figure S4. Detail of the face-to-face stacked $\pi \cdots \pi$ interactions [centroid-centroid distance of $c a .3 .7 \AA]$ between pyridine rings of $\left[\left\{\mathrm{RuCl}_{4}(\mathrm{py})\right\}_{2}(\mu-\mathrm{O})\right]^{4-}$ anions in the crystal of 5. Color code: orange, Ru; green, $\mathrm{Cl}$; red, O; blue, N; white, C.

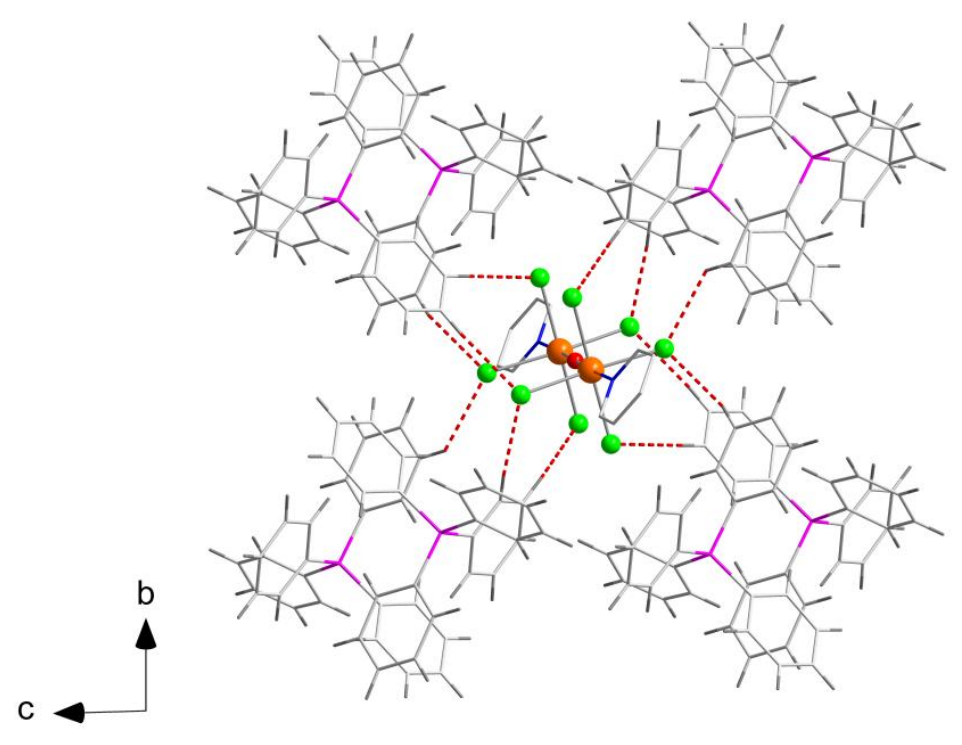

Figure S5. View along the $a$ axis of the $\mathrm{C}-\mathrm{H} \cdots \mathrm{Cl}$ interactions between $\mathrm{PPh}_{4}{ }^{+}$cations and one $\left[\left\{\mathrm{RuCl}_{4}(\mathrm{py})\right\}_{2}(\mu-\mathrm{O})\right]^{4-}$ anion in the crystal of $\mathbf{5}$. Color code: orange, $\mathrm{Ru}$; green, $\mathrm{Cl}$; P, pink; red, O; blue, N; white, C; grey, $\mathrm{H}$. 


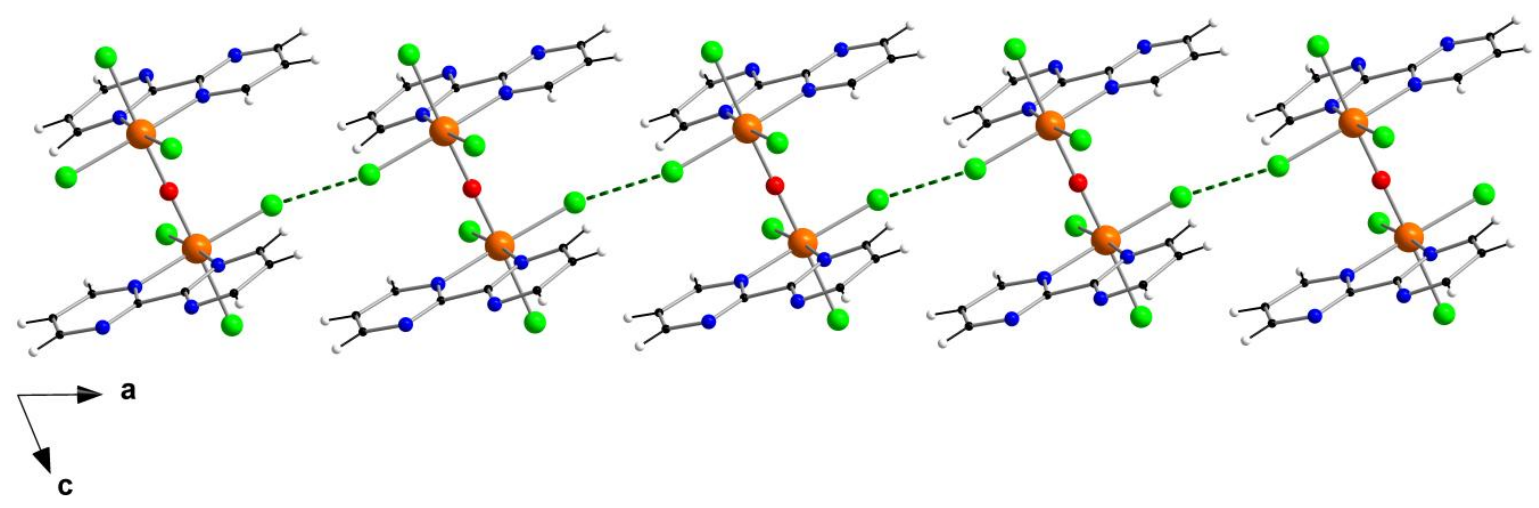

Figure S6. Detail of the one-dimensional motif generated through intermolecular $\mathrm{Cl} \cdots \mathrm{Cl}$ interactions (dashed green lines) between neighboring $\left[\left\{\mathrm{RuCl}_{3}(\mathrm{bpym})\right\}_{2}(\mu-\mathrm{O})\right]$ complexes in the crystal of $\mathbf{6}$. Crystallization water molecules have been omitted for clarity. Color code: orange, $\mathrm{Ru}$; green, $\mathrm{Cl}$; red, O; blue, N; black, C; white, $\mathrm{H}$. 


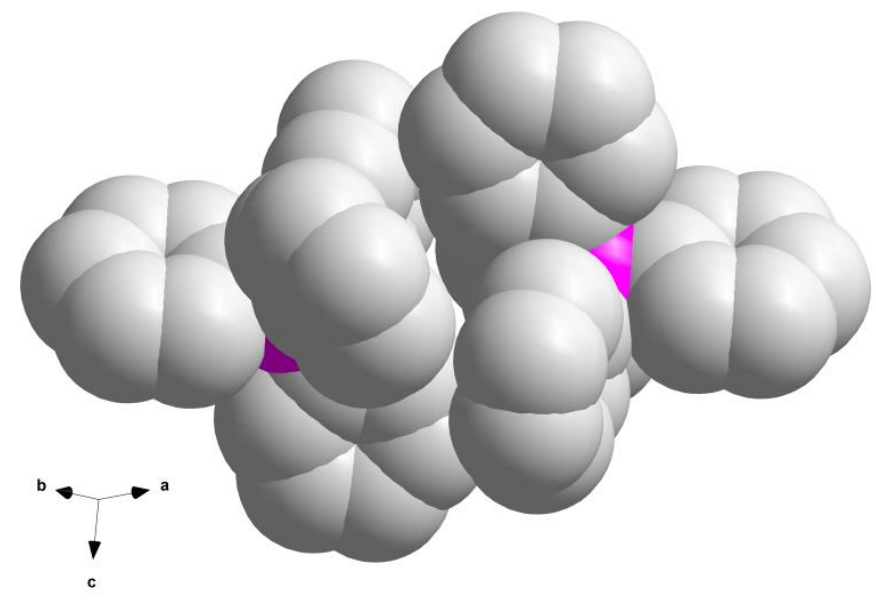

Figure S7. Sextuple phenyl embrace (SPE) supramolecular conformation of the $\mathrm{PPh}_{4}{ }^{+}$ cations (space-filling model) in $\mathbf{2}$. $\mathrm{H}$ atoms have been omitted for clarity. Color code: $\mathrm{P}$, pink; grey, C. [Symmetry code: $(b)=-x+1,-y-1,-z+1]$.

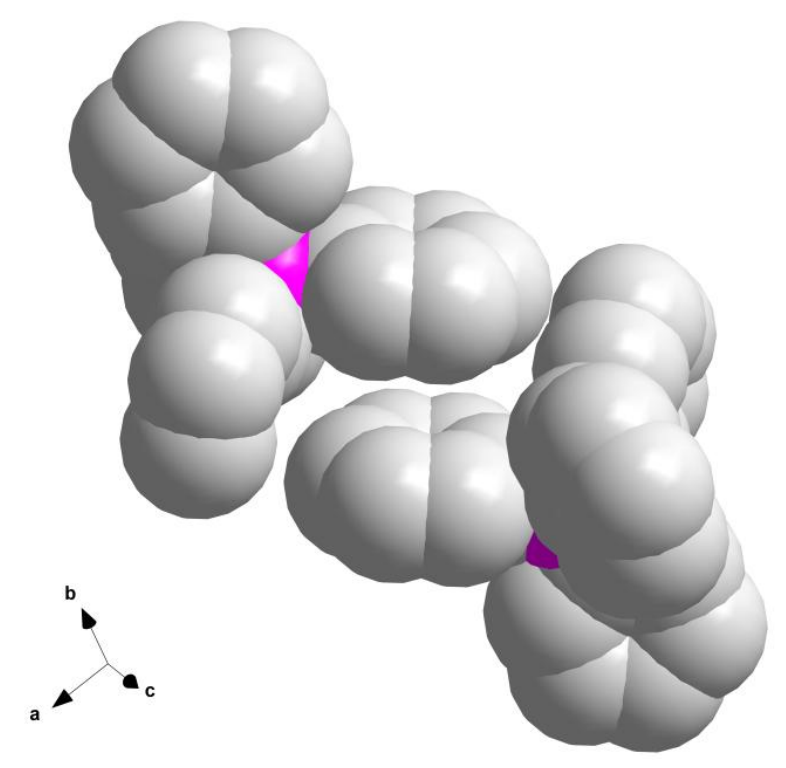

Figure S8. Quadruple phenyl embrace (PQPE) motif of the $\mathrm{PPh}_{4}{ }^{+}$cations (space-filling model) in 2. $\mathrm{H}$ atoms have been omitted for clarity. Color code: $\mathrm{P}$, pink; grey, $\mathrm{C}$. [Symmetry code: $(\mathrm{c})=-\mathrm{x}+1,-\mathrm{y},-\mathrm{z}]$. 


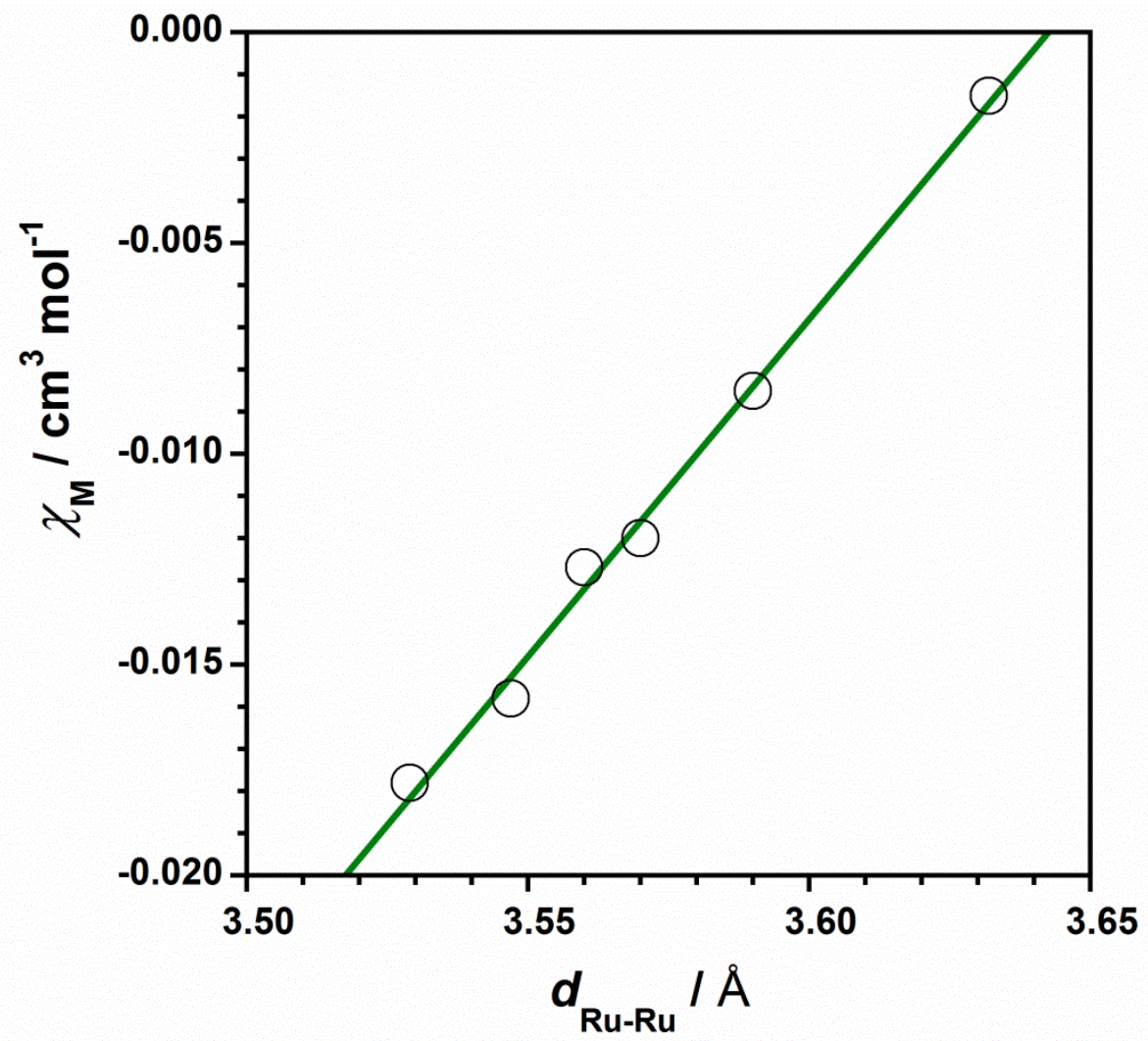

Figure S9. Plot of the molar magnetic susceptibility $\left(\chi_{\mathrm{M}}\right)$ versus the intramolecular Ru$\mathrm{Ru}$ distance for 1-6. The solid line represents the best-fit of the experimental data. 

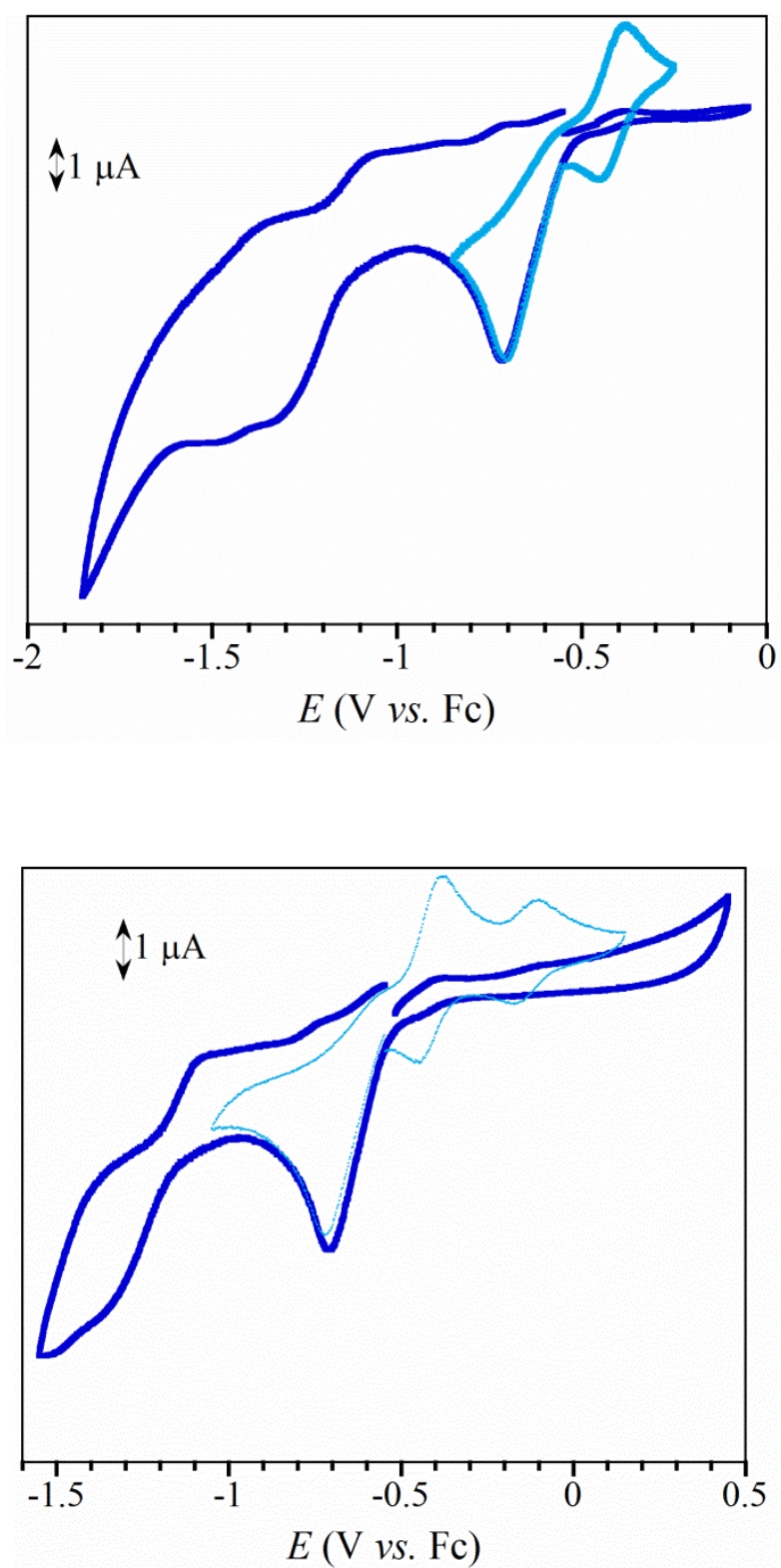

Figure S10. Cyclic voltammograms of 2 (top) and 3 (bottom) in dry dmf (0.1 $\mathrm{M}$ $\mathrm{NBu}_{4} \mathrm{PF}_{6}$ ) at $25{ }^{\circ} \mathrm{C}$ and $200 \mathrm{mV} \mathrm{s}^{-1}$. The pale blue curves show the cyclic voltammograms of the oxidation waves when the potential is scanned through the first reversible reduction waves. 
Table S7. Electrochemical potential data for $2-6^{a, b}$

$$
\begin{array}{lccccc} 
& \mathbf{2}^{\mathbf{i}} & \mathbf{3}^{\mathbf{i}} & \mathbf{4}^{\mathbf{i}} & \mathbf{5}^{\mathbf{i}} & \mathbf{6} \\
E_{1}\left(\Delta E_{1}\right) & -0.75 & -0.77 & -0.77 & -0.80 & -0.61(-92) \\
E_{2}\left(\Delta E_{2}\right) & -1.34 & -1.60 & -1.59 & -1.68 & -0.78(-97) \\
E_{3}\left(\Delta E_{3}\right) & - & - & - & - & -1.05(-138) \\
E_{4}\left(\Delta E_{4}\right) & - & - & - & - & -1.26(-144) \\
E_{5}\left(\Delta E_{5}\right) & - & - & - & - & +0.17(-75)
\end{array}
$$

${ }^{a}$ Formal potentials are defined as the half-wave potentials for reversible waves or as the cathodic potentials for the irreversible (i) reduction waves (in V). $\Delta E_{n}$ are the values of the peak-to-peak separations (in $\mathrm{mV}$ ).

${ }^{b}$ In dry DMF and at room temperature $\left(0.1 \mathrm{M} \mathrm{NBu}_{4} \mathrm{PF}_{6}\right)$.

Table S8. Experimental absorption spectroscopic data for 2-6 ${ }^{a, b}$

$\begin{array}{cccccc}\lambda_{\mathrm{n}} / \mathrm{nm} & \mathbf{2} & \mathbf{3} & \mathbf{4} & \mathbf{5} & \mathbf{6} \\ \left(\varepsilon \cdot 10^{-5} / \mathrm{M}^{-1} \mathrm{~cm}^{-1}\right) & & & & & \\ \lambda_{1}\left(\varepsilon_{1}\right) & 398 & 399 & 399 & 437 & 358 \\ & (2.69) & (2.35) & (2.31) & (1.31) & (1.67) \\ \lambda_{2}\left(\varepsilon_{2}\right) & 493 & 495 & 495 & 501 & 397 \\ & (0.86) & (0.76) & (0.75) & (0.76) & (2.84) \\ \lambda_{3}\left(\varepsilon_{3}\right) & - & - & - & - & 498 \\ & & & & & (1.84)\end{array}$

${ }^{a}$ In dry DMF and at room temperature.

${ }^{b}$ Values of the absorption maxima. The extinction coefficient values are given in parenthesis. 

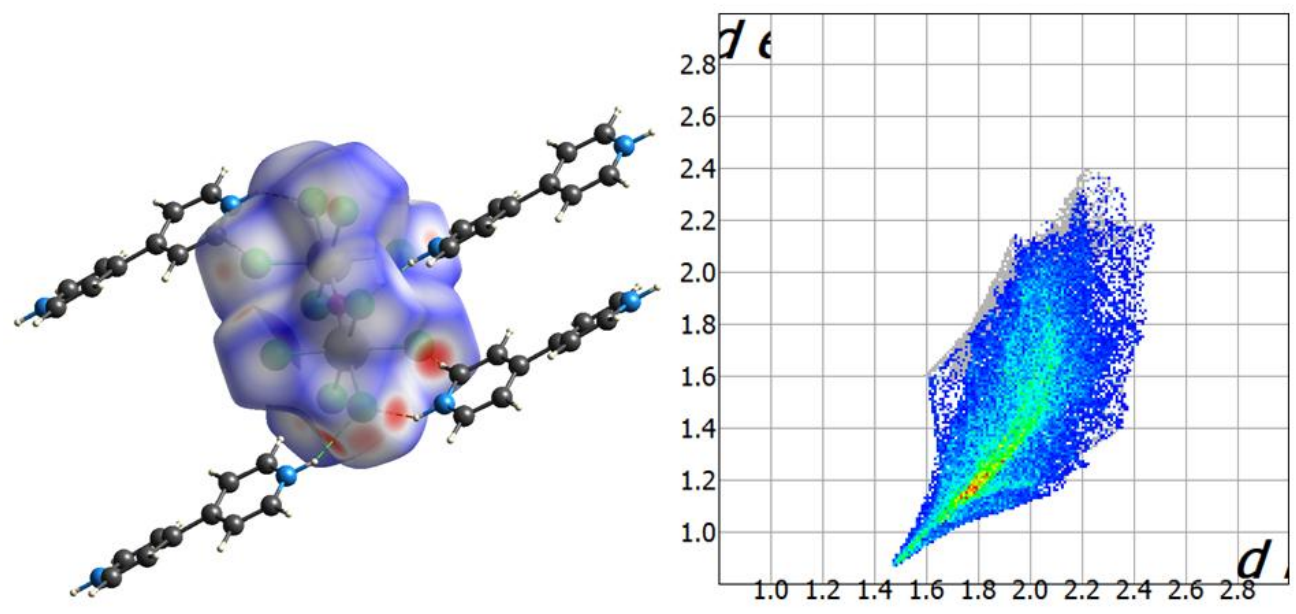

Figure S11. Hirshfeld surface mapped with $d_{\text {norm }}$ function (left) and fingerprint plot (right) for compound 1. Intermolecular $\mathrm{Cl} \cdots \mathrm{H}$ contacts are approximatelly the $90 \%$ of the complete fingerprint.
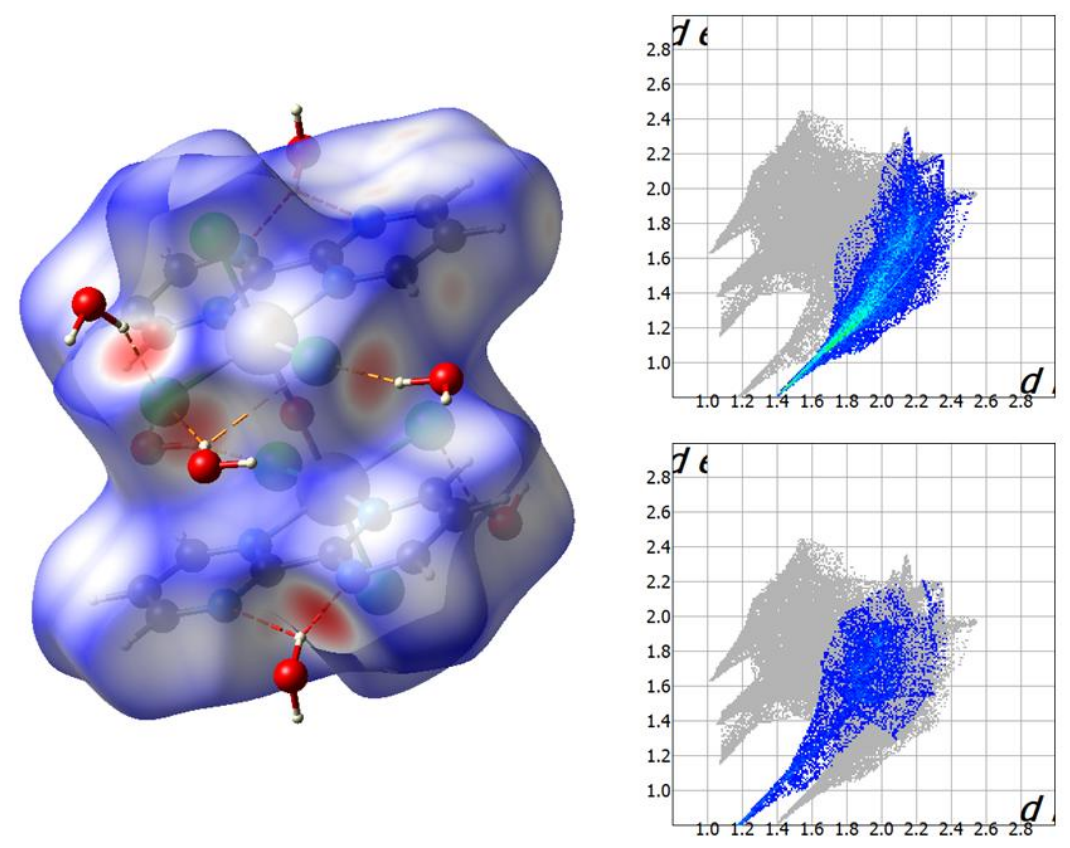

Figure S12. Hirshfeld surface mapped with $d_{\text {norm }}$ function (left) and fingerprint plots (right) for compound 6. Intermolecular $\mathrm{Cl} \cdots \mathrm{H}$ (righ, top) and $\mathrm{N} \cdots \mathrm{H}$ (right, bottom) contacts are highlighted from the full fingerprint. 